\title{
Meaningful Family Relationships: Neurocognitive Buffers of Adolescent Risk Taking
}

\author{
Eva H. Telzer ${ }^{1}$, Andrew J. Fuligni ${ }^{2}$, Matthew D. Lieberman², \\ and Adriana Galván ${ }^{2}$
}

\begin{abstract}
Discordant development of brain regions responsible for cognitive control and reward processing may render adolescents susceptible to risk taking. Identifying ways to reduce this neural imbalance during adolescence can have important implications for risk taking and associated health outcomes. Accordingly, we sought to examine how a key family relationship—family obligation—can reduce this vulnerability. Fortyeight adolescents underwent an fMRI scan during which they completed a risk-taking and cognitive control task. Results sug-
\end{abstract}

\section{INTRODUCTION}

Adolescence is a time of heightened vulnerability for risk taking, impulsivity, and reckless behavior. From childhood to adolescence, there is a significant increase in risky behaviors such as experimentation with and abuse of drugs and alcohol, unsafe sexual practices, dangerous driving, and violent and reckless behaviors (Casey, Getz, \& Galvan, 2008; Steinberg, 2008; Dahl, 2004). Risk taking underlies many behavioral and health problems that contribute to the public health burden during the adolescent period, contributing to a dramatic increase in morbidity and mortality rates (Miniño, 2010; Dahl, 2004; Chambers, Taylor, \& Potenza, 2003; Arnett, 1992). Given the serious consequences of adolescent risk taking, it is imperative to identify potential protective factors that may reduce vulnerability for health-compromising risk taking.

Risk-taking behaviors show a typical developmental trajectory, such that they are low in childhood, increase around puberty, peak in mid adolescence, and then decrease in adulthood (Figner, Mackinlay, Wilkening, $\&$ Weber, 2009). The dual systems model of adolescent risk taking proposes that the balance between affective and cognitive processes underlies this developmental trajectory in risk taking (Somerville, Jones, \& Casey, 2010; Steinberg, 2008). Functional neuroimaging research shows that reward sensitivity shows curvilinear developmental patterns, peaking in mid adolescence, whereas

\footnotetext{
${ }^{1}$ University of Illinois, Urbana-Champaign, ${ }^{2}$ University of California, Los Angeles
}

gest that adolescents with greater family obligation values show decreased activation in the ventral striatum when receiving monetary rewards and increased dorsolateral PFC activation during behavioral inhibition. Reduced ventral striatum activation correlated with less real-life risk-taking behavior and enhanced dorsolateral PFC activation correlated with better decision-making skills. Thus, family obligation may decrease reward sensitivity and enhance cognitive control, thereby reducing risk-taking behaviors. cognitive control capacities demonstrate protracted maturation, showing improvements well into adulthood (Luna, Padmanabhan, \& O'Hearn, 2010; Galvan et al., 2006; Bunge, Dudukovic, Thomason, Vaidya, \& Gabrieli, 2002). For example, adolescents show heightened ventral striatal (VS) activation to rewarding stimuli compared with both children and adults, and this reactivity is associated with increased reward-seeking behaviors (Galvan, Hare, Voss, Glover, \& Casey, 2007; Galvan et al., 2006). In contrast, adolescents show less activation in prefrontal regions compared with adults when making risky decisions, and this dampened activation is associated with greater risk taking (Eshel, Nelson, Blair, Pine, \& Ernst, 2007). Particularly during states of heightened emotional arousal, reward activation may outweigh or override cognitive control capacities, resulting in riskier choices (Chein, Albertm, O’Brien, Uckert, \& Steinberg, 2010; Figner et al., 2009; Steinberg, 2008). Immature cognitive control development relative to the reward system may hinder appropriate evaluation of risk and bias youth toward risky decisions.

The dual systems model of adolescent risk taking has received considerable attention and offers a promising way to understand why adolescents may be vulnerable to risk taking. However, scholars have recently begun to refine the model, highlighting the complexity of developmental brain function and recognizing the importance of considering how social contexts may influence the development of these neural systems (Crone \& Dahl, 2012; Pfiefer \& Allen, 2012). Risk taking does not occur in a social vacuum, and it is critical to examine how neural mechanisms interact with fundamental social processes during 
adolescence. Thus, it is important to understand individual differences in the developmental trajectories of affective and cognitive brain function.

The changing nature of family relationships during the adolescent years can have significant implications for risk taking and associated health consequences, such as substance use and externalizing problems (Warner et al., 2006; Gfoerer \& de la Rosa, 1993). Family obligation-the importance of spending time with the family, high family unity, family social support, and interdependence for daily activities (Fuligni, 2001; Cuellar, Arnold, \& Gonzalez, 1995) - is a key aspect of family relationships that may have significant consequences for adolescents' health. Family obligation and family pride have been associated with reduced likelihood and delayed onset of drug use and lower rates of externalizing problems (German, Gonzales, \& Dumka, 2009; Romero \& Ruiz, 2007; Ramirez \& de la Cruz, 2003; Unger et al., 2002; Kaplan, NapolesSpringer, Stewart, \& Perez-Stable, 2001; Gil, Wagner, \& Vega, 2000; Vega, Zimmerman, Warheit, Apospori, \& Gil, 1993). Participating in a daily routine such as family assistance that is meaningful with respect to group goals and values leads to enhanced well-being and builds confidence (Weisner, Matheson, Coots, \& Bernheimer, 2005). Indeed, we have found that adolescents who assist their family more feel that they are fulfilling important roles within their family and have higher levels of daily happiness (Telzer \& Fuligni, 2009). Moreover, neuroimaging research shows that family assistance is associated with activation in neural regions responsive to reward (Telzer, Masten, Berkman, Lieberman, \& Fuligni, 2010). Together, these studies suggest that family obligation is associated with feelings of value, meaningfulness, and intrinsic reward, which may deter adolescents from engaging in health compromising risky behavior.

Family obligation may reduce risk taking because it is a meaningful activity that increases adolescents' motivation to control their own impulses and desires for the sake of their family as well as provides opportunities to practice engaging in self-control. For example, adolescents who value family obligation report greater negative consequences for engaging in risky behavior because risk taking reflects poorly upon their family (German et al., 2009). Therefore, the negative consequences of risk taking may be more consequential for these youth, and so risk taking becomes comparatively less rewarding. Risk taking is amplified during states of affective arousal among adolescents, such as in the presence of peers (Chein et al., 2010; Figner et al., 2009; Gardner \& Steinberg, 2005). In contrast, during "cold" deliberative states of decision-making, heightened risk taking is not always found among adolescents (Figner et al., 2009). Therefore, family obligation values may decrease the affective nature of risk taking, reducing reward sensitivity during risk. In the absence of heightened arousal, cognitive control capacities may come on-line and reduce risky decision-making. Likewise, adolescents who value family obligation may be more motivated to engage in self-control to avoid risky behaviors. Indeed, scholars refining the dual systems model suggest that cognitive control capacities are not necessarily immature during adolescence, but, rather, there is a great deal of flexibility, and the degree to which adolescents engage in cognitive control is strongly influenced by the motivational salience of the context (Crone \& Dahl, 2012). Thus, reward sensitivity and self-control may be altered by adolescents' motivation to avoid risk taking.

It is also possible that family obligation increases selfcontrol and decreases reward sensitivity because of practice and learning. For example, adolescents who value family obligation often put the needs of their family before their own (Fuligni, Tseng, \& Lam, 1999), which may help them exert self control to make decisions that benefit their family. Moreover, family obligation values stress the importance of considering future family needs (Fuligni et al., 1999), which may increase adolescents' goal directed behavior and ability to plan ahead. Indeed, adolescents who report greater family obligation values show enhanced activation in neural regions implicated in cognitive control (e.g., IPFC), suggesting that decisions to assist one's family are not automatic but require adolescents to engage in selfcontrol (Telzer, Masten, Berkman, Lieberman, \& Fuligni, 2011). Practice engaging in cognitive control is associated with longitudinal increases in IPFC activation among adolescents, suggesting that frontal activation can be enhanced through practice (Jolles, van Buchem, Rombouts, \& Crone, 2012). Family obligation may be one way to enhance self-control.

In the current study, we sought to answer two key questions. First, how does family obligation relate to neural markers of risk taking? We predicted that family obligation would be associated with decreased VS activation during a risk-taking task and increased lateral PFC activation during a behavioral inhibition task. Moreover, because family obligation provides motivation for adolescents to engage in self-control and may increase the perceived negative consequences of risk taking, we tested whether family obligation would be uniquely protective against risk taking, above and beyond the effects of more general levels of emotional cohesion to the family. Therefore, we examined how family obligation and family cohesion and support simultaneously predict neural responses to reward and cognitive control. Second, does activation in the VS and lateral PFC predict adolescents' real-life risk taking behavior and decision-making skills? Scholars refining the dual systems model have stressed the importance of linking neural activations to relevant real-life behaviors. Recent advances in neuroimaging have shown that neural response to risk taking and decisionmaking are associated with increased likelihood of risktaking behaviors among adolescents (Bjork, Smith, Chen, \& Hommer, 2010; Eshel et al., 2007; Galvan et al., 2007). In the current study, we predicted that neural regions that are sensitive to family obligation during reward (e.g., the VS) will relate to less risk taking behavior, and neural 
regions sensitive to family obligation during cognitive control (e.g., IPFC) will relate to better decision-making skills.

We focus on the links between family obligation and neural markers of risk taking among adolescents from Mexican backgrounds. The family may be an especially important protective factor for Latino youth (Warner et al., 2006; Gfoerer \& de la Rosa, 1993; de la Rosa, 1988) who place particular value on family obligation and spend twice as much time helping their family each day compared with their European American peers (Telzer \& Fuligni, 2009; Fuligni, 2001; Cuellar et al., 1995; García Coll \& Vázquez García, 1995; Suárez-Orozco \& Suárez-Orozco, 1995). Indeed, staying out of trouble is an important aspect of the family obligations of youth from Mexican backgrounds (Suárez-Orozco \& Suárez-Orozco, 1995; Vega et al., 1993).

\section{METHODS}

\section{Participants}

Forty-eight adolescents from Mexican backgrounds participated in an fMRI scan. Participants ranged in age from 14 to 16.5 years ( $M_{\text {age }}=15.23 ; 21$ boys, 27 girls). All but one participant spoke and read English fluently. For the Spanish-speaking participant, all tasks and questionnaire measures were described and administered in Spanish. Participants completed written consent and assent in accordance with University of California-Los Angeles's institutional review board.

\section{Questionnaire Measures}

\section{Family Obligation Values}

Participants used a 5-point scale ( $1=$ almost never to 5 = almost always) to respond to 12 questions describing their expectations for how often they feel they should assist with household tasks and spend time with their family (e.g., "help take care of your brothers and sisters," "eat meals with your family," and "spend time with your family on weekends"; Fuligni et al., 1999).

\section{Family Cohesion and Support}

Family cohesion and support were measured using the parent subscale of the Inventory of Parent and Peer Attachment (Armsden \& Greenberg, 1987). Using a 5-point scale $(1=$ almost never to $5=$ almost always), participants answered 19 questions indicating to what extent they felt close to and supported by their parents. Example items include, "I could count on my parents when I needed to talk" and "I trusted my parents."

\section{Risk-taking Behavior}

A modified version of the Adolescent Risk-taking Scale (Alexander et al., 1990) was used to measure how often adolescents engage in risky behaviors. Adolescents re- sponded to nine items using a 4-point scale (never, once or twice, several times, and many times) to indicate the frequency with which they have engaged in the following behaviors: raced on a bike or boat, did something risky or dangerous on a dare, broke a rule that their parents set just for the thrill of seeing if they could get away with it, stole or shoplifted, slipped out at night while their parents thought they were asleep, willingly rode in a car with someone who was a dangerous driver, tagged or defaced public property, drove in a car without wearing a seatbelt, and had sex without using protection.

\section{Decision-making Competence}

The Flinders Adolescent Decision-making Questionnaire (Mann, Harmoni, \& Power, 1989) was used to examine adolescents' decision-making strategies. Adolescents responded using a 4-point scale $(1=$ not at all true of me to $4=$ almost always true of $m e$ ) to six items assessing decision-making vigilance (e.g., "I take a lot of care before I make my choice" and "I like to think about a decision before I make it") and six items assessing decision-making avoidance (e.g., "I avoid making decisions" and "I put off making decisions"). The avoidance items were reverse scored, and the two subscales were averaged to create a measure of decision-making competence.

\section{fMRI Paradigms}

Risk-taking Task

To examine neural sensitivity to risk, participants completed the Balloon Analogue Risk Task (BART; Lejuez et al., 2002). Importantly, behavioral performance on the BART correlates with real-life risk behaviors such as adolescent smoking, sexual promiscuity, addiction, and drug use (Bornovalova et al., 2009; Lejuez, Aklin, Zvolensky, \& Pedulla, 2003), suggesting that this task provides a scannercompatible proxy for measuring real-world behaviors.

On each trial of the task, participants are shown a virtual red-colored balloon and given the option to inflate the balloon, which can either grow larger or explode (Figure 1). The larger the balloon is inflated, the greater the monetary reward but the higher the probability of explosion. Participants press one of two buttons to either inflate (pump) the balloon or to "cash-out." Each trial begins with the presentation of a balloon and ends when the balloon either explodes or the participant cashes out. The participant receives a payoff (25 cents) for each pump on which the balloon is successfully inflated and can stop inflating the balloon at any point and keep the accumulated payoff. If the balloon explodes, the participant receives no payoff for that trial, but earnings from previous trials are unaffected. The number of inflations before explosion is varied probabilistically according to a Poisson distribution. This pattern models the unpredictable rewards and punishments that characterize real-world 
Figure 1. The BART has three types of conditions: (A) explosions, in which the participant inflated the red balloon resulting in an explosion; (B) cash outs, in which the participant inflated the red balloon and successfully cashed out; and (C) control, in which the participant inflated white balloons, which are not associated with a monetary reward.

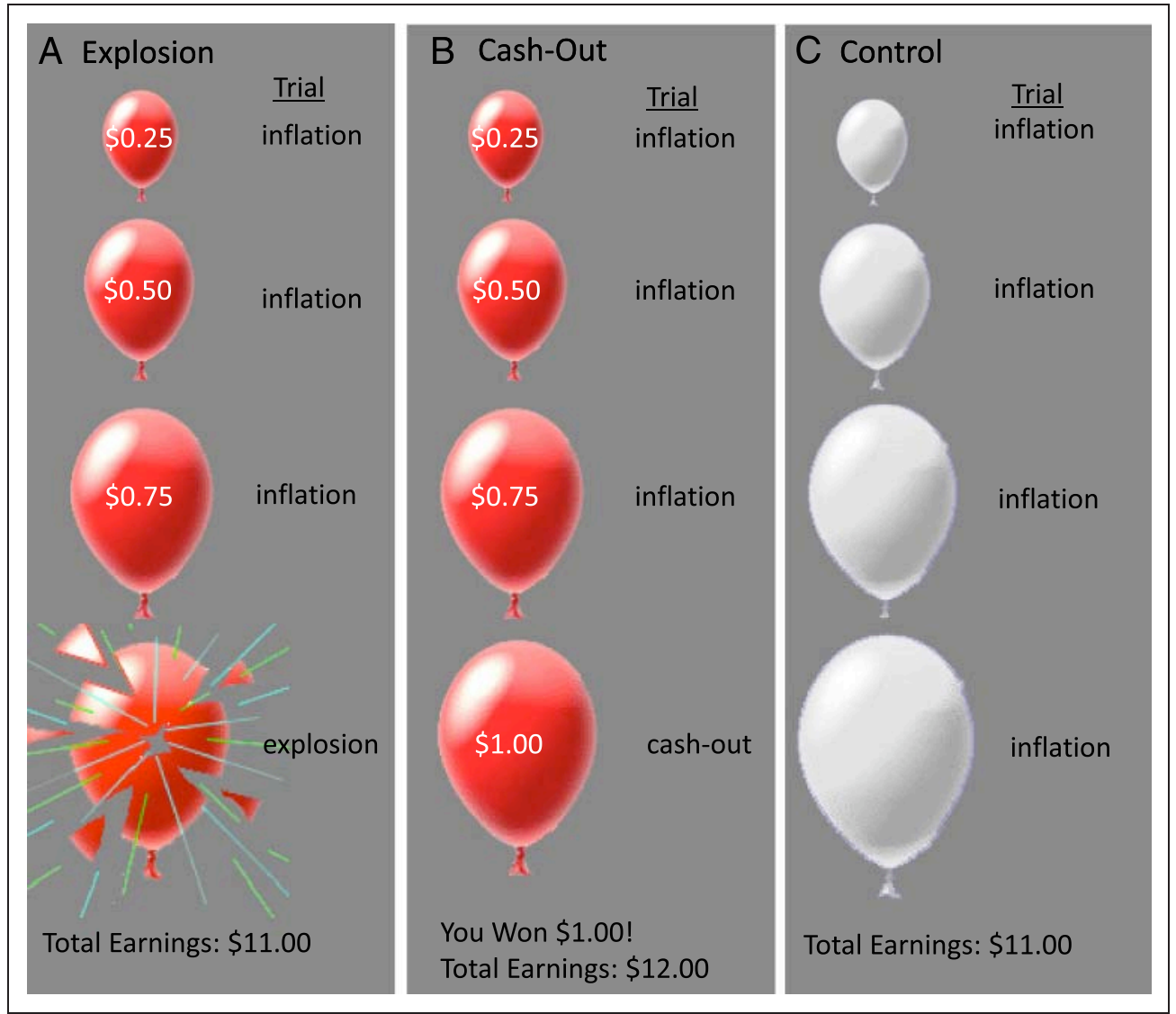

risky behaviors. As pumping progresses during a trial, explosion probability increases exponentially. The explosion point of each balloon was drawn from a uniform distribution from 1 to 12 pumps. In addition, 25\% of the balloons were white and were not associated with a reward or possible explosion and provided a control for the visual and motor aspects of pumping. Participants were instructed to "pump" the white balloons until they disappeared. White balloons did not explode but inflated according to the same distribution as the red balloons. After each pump, the balloon image disappeared (1-3 sec, variable duration) until the outcome was displayed: a larger balloon or an exploded one. At the end of each trial, the screen was blank for a varying duration (1-12 sec, average $4 \mathrm{sec})$. The task was self-paced and was performed during one 9-min run. Because the task was determined in length by time, the total number of balloons varied across participants. Participants received their total earnings at the end of the task.

\section{Cognitive Control Task}

Participants completed a standard go/no-go (GNG) task as a functional localizer to target cognitive control-related brain function. Participants were presented with a series of rapid trials ( $1 \mathrm{sec}$ each), each displaying a single letter, and were instructed to respond with a button press as quickly as possible to all letters except for $\mathrm{X}$. The $\mathrm{X}$ occurred on $25 \%$ of trials. Thus, participants develop a pre- potent response to press (go) upon stimulus onset and must inhibit the go response on X trials (no-go). Response inhibition was operationalized as successful no-go trials (i.e., overriding the prepotent go response) compared with go trials. Participants completed five blocks, each of which contained an average of 10 no-go trials and 30 go trials. The intertrial interval was jittered according to a random gamma distribution $(M=0.75 \mathrm{sec})$. Each block ( 40 trials and intertrial intervals) lasted $70 \mathrm{sec}$, and blocks were separated by 12 -sec rest periods.

\section{fMRI Data Acquisition and Analysis}

\section{fMRI Data Acquisition}

Imaging data were collected using a 3-T Siemens Trio MRI scanner. The tasks were presented on a computer screen, which were projected through scanner-compatible goggles. The BART task consisted of 270 functional T2*weighted EPIs and the GNG task consisted of 200 images (slice thickness $=4 \mathrm{~mm}, 34$ slices, repetition time $=$ $2 \mathrm{sec}$, echo time $=30 \mathrm{msec}$, flip angle $=90^{\circ}$, matrix $=$ $64 \times 64$, field of view $=200 \mathrm{~mm}$, voxel size $=3 \times 3 \times$ $4 \mathrm{~mm}^{3}$ ). A T2*-weighted, matched-bandwidth (MBW), high-resolution, anatomical scan and MPRAGE scan were acquired for registration purposes (repetition time $=2.3$, echo time $=2.1$, field of view $=256$, matrix $=192 \times 192$, sagittal plane, slice thickness $=1 \mathrm{~mm}, 160$ slices). The 
orientation for the MBW and EPI scans was oblique axial to maximize brain coverage.

\section{fMRI Data Preprocessing and Analysis}

Analyses were performed using FMRIB Software Library (FSL) 4.1 .6 (www.fmrib.ox.ac.uk/fsl). All images were skull-stripped using FSL BET. The images were realigned to compensate for small head movements (Jenkinson, Bannister, Brady, \& Smith, 2002). No participants exceeded $>2 \mathrm{~mm}$ in translational or $2^{\circ}$ in rotational movement. Data were smoothed using a 5-mm FWHM Gaussian kernel to increase the signal-to-noise ratio and filtered in the temporal domain using a nonlinear high-pass filter (100-sec cutoff). EPI images were registered to the MBW, then to the MPRAGE, and finally into standard MNI space (MNI152, T1 $2 \mathrm{~mm}$ ) using linear registration with FSL FLIRT.

One general linear model was defined for the BART, which included multiple regressors for each event type: color pumps (i.e., pumps for red balloons), control pumps (i.e., pumps for white balloons), cash outs, and explosions. For the color pumps, we analyzed the adjusted pumps, which represents the number of pumps on balloons that did not explode. This is preferable to examining pumps on balloons that did explode, because the number of pumps is necessarily constrained on balloons that explode (Lejuez et al., 2002). Color pumps, control pumps, cash outs, and explosions were modeled with a parametric regressor that tested for the linear relationship between brain activation and the magnitude of pumps, reward, or loss. We used pump number as a parametric modulator, with each pump in a trial assigned a weight that increased linearly across pumps within a trial. On cash-out trials and explosions, this number represented how many pumps occurred before the cash out or explosion. The number of pumps was demeaned by subtracting the mean number of pumps from each pump number within the trial. Because the task was self-paced, the duration of each trial represented the RT for that trial. Null events, consisting of the jittered intertrial intervals, were not explicitly modeled and therefore constituted an implicit baseline. Group level contrasts of interest included Pumps $>$ Control (which is the contrast of color pumps vs. white pumps), Cash Outs $>$ Baseline, and Explosions $>$ Baseline.

For the GNG task, one general linear model was defined, which included multiple regressors for each event type: successful go trials, successful no-go trials, and false alarms. Events were modeled with a 1-sec duration. The rest periods and jittered intertrial intervals were not explicitly modeled and therefore served as an implicit baseline. Our group level contrast of interest was no-go > go. For both tasks, temporal derivatives and motion parameters were included as covariates of no interest for all regressors.

The FSL FEAT package was used for statistical analysis. Regressors of interest were created using a stick function of the event duration at the onset time of each trial with a canonical (double-gamma) hemodynamic response function. A group-level analysis was performed using the FMRIB Local Analysis of Mixed Effects module in FSL (Beckmann, Jenkinson, \& Smith, 2003). Thresholded $Z$-statistic images were prepared to show clusters determined by a corrected, cluster-forming threshold of $Z>$ 2.3 and an extent threshold of $p<.05$ familywise error corrected using the Theory of Gaussian Random Fields (Poline, Worsley, Evans, \& Friston, 1997). Outliers were deweighted in the multisubject statistics using mixture modeling (Woolrich, 2008). To examine correlations between family obligation values and reward sensitivity on the BART and cognitive control on the GNG, family obligation scores were entered as a regressor in the contrasts of interest. For visualization, statistical maps of all analyses were projected onto a study-specific average brain of the participants.

\section{RESULTS}

\section{Behavioral Results}

Means, standard deviations, ranges, and correlations among all questionnaire measures and behavioral performance on the BART and GNG are displayed in Table 1. There were no age or gender differences in any of the study variables.

Table 1. Descriptives of All Study Variables

\begin{tabular}{|c|c|c|c|c|c|c|c|c|}
\hline & \multirow[b]{2}{*}{ Mean (SD) } & \multirow[b]{2}{*}{ Range } & \multicolumn{6}{|c|}{ Correlations } \\
\hline & & & 1 & 2 & 3 & 4 & 5 & 6 \\
\hline 1. Family obligation & $3.3(0.61)$ & $2.3-5.0$ & 1 & $.30 *$ & -.19 & .21 & $-.31 *$ & .07 \\
\hline 2. Family cohesion & $2.9(1.1)$ & $1.2-5.0$ & & 1 & $-.29 *$ & .16 & -.26 & -.10 \\
\hline 3. Risky behavior & $1.7(0.48)$ & $1-2.1$ & & & 1 & -.07 & .21 & .08 \\
\hline 4. Decision-making & $3.0(0.44)$ & $2.1-3.8$ & & & & 1 & -.23 & .09 \\
\hline 5. Mean pumps BART & $3.8(1.1)$ & $1.9-6.2$ & & & & & 1 & .14 \\
\hline 6. \% False alarms GNG & $16.8(12.9)$ & $0-60$ & & & & & & 1 \\
\hline
\end{tabular}


Table 2. Neural Regions that Correlated with (A) Gender and Risk Taking and (B) Age and Response Inhibition

\begin{tabular}{lllllll}
\hline Anatomical Region & $x$ & $y$ & $z$ & $\operatorname{Max} Z$ & $k$ \\
\hline
\end{tabular}

A. Gender Differences in Brain Activation on the BART

Cash outs $>$ baseline, females $>$ males

$\begin{array}{lrrrrr}\text { R Cuneus } & 8 & -102 & -2 & 4.0 & 1101 \\ \text { L Fusiform } & -26 & -54 & -10 & 3.6 & 775 \\ \text { L Cuneus } & -22 & -94 & 18 & 3.8 & 687 \\ \text { R Fusiform } & 38 & -46 & -24 & 4.1 & 480\end{array}$

Pumps $>$ control, males $>$ females

$\begin{array}{lrrrrr}\text { R Precuneus } & 6 & -60 & 44 & 3.9 & 691 \\ \text { L BA 3,1,2 } & -16 & -26 & 68 & 4.1 & 520\end{array}$

B. Age Difference in Brain Activation on GNG no-go $>$ go

\begin{tabular}{lrrrrr} 
L Cuneus & -6 & -74 & 18 & 4.3 & 1272 \\
R pSTS & 44 & -68 & 26 & 3.8 & 688 \\
L Precuneus & -2 & -68 & 52 & 4.2 & 622 \\
L pSTS & -50 & -52 & 6 & 3.6 & 610 \\
R DLPFC & 36 & 18 & 44 & 3.5 & 542 \\
\hline
\end{tabular}

$\mathrm{L}$ and $\mathrm{R}$ refer to left and right hemispheres; $x y$ and $z$ refer to MNI coordinates; Max $Z$ refers to the $z$ score at those coordinates (local maxima); $k$ refers to the number of voxels in each significant cluster. All regions are listed at cluster-forming threshold of $Z>2.3$ and an extent threshold of $p<.05$ corrected using the Theory of Gaussian Random Fields. pSTS $=$ posterior STS

\section{$B A R T$}

Participants completed 20.5 total red balloons on average (range $=13-34$ balloons). On average, participants inflated $3.79(S D=1.05)$ pumps per red balloon, exploded $33.83 \%(S D=9.7 \%)$ of red balloons, and success- fully cashed out on $64.36 \%(S D=10.34 \%)$ of red balloons. Those with higher average pumps completed fewer total balloons on average $(r=-.34, p<.01)$. Participants took significantly longer to cash out $(M=0.91 \mathrm{sec}$, $S D=.31 \mathrm{sec})$ than to inflate balloons $[M=0.75 \mathrm{sec}, S D=$ $.26 \mathrm{sec}, t(47)=3.69, p=.001]$ and earned a total of $\$ 15.64(S D=\$ 4.01)$ on average (range $=\$ 8.25-\$ 26.75)$. To examine how family obligation relates to behavioral performance on the BART, we conducted regression analyses controlling for gender and age. Adolescents with greater family obligation values had lower mean pumps $(\beta=$ $-.31, p<.05)$, suggesting a lower propensity toward risky, reward-sensitive behavior. When we entered family cohesion into the same regression model, family obligation is marginally associated with mean pumps $(\beta=-.27$, $p=.07$ ) and family cohesion is not related to mean pumps $(\beta=-.13, p=.38)$. Family obligation was not related to the percentage of balloons that were cashed or exploded or to mean RT. There were no age or gender differences on behavioral performance on the BART.

\section{GNG}

On average, participants successfully inhibited 83.23\% ( $S D=12.92 \%$ ) of the no-go trials (i.e., withheld the button press to the no-go trials), ranging from $40 \%$ to $100 \%$. Participants' mean RT was significantly faster to false alarms $(M=.36 \mathrm{sec}, S D=.06 \mathrm{sec})$ than to successful go trials $[M=427 \mathrm{msec}, S D=43.83 \mathrm{msec} ;(44)=8.15, p<.001]$. Family obligation values were not related to false alarms or mean RT. There were no age or sex differences on behavioral performance on the GNG.

\section{fMRI Results}

\section{Main Effects on the BART}

In whole-brain analyses, we examined neural activation to pumps, cash outs, and explosions. As shown in Table 2A and Figure 2A, the contrast used to examine activation
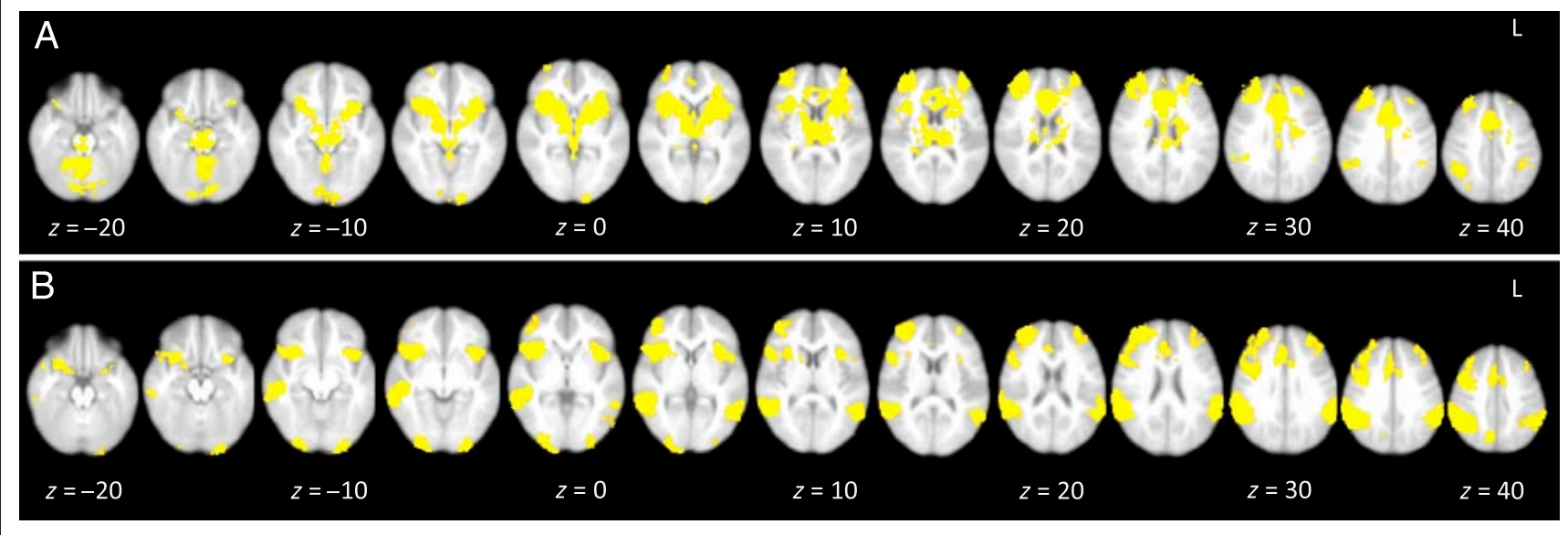

Figure 2. Main effects on the (A) BART to pumps $>$ control and (B) GNG to no-go $>$ go. 
associated with increasing pumps (Pumps $>$ Control) revealed activation in the bilateral VS, bilateral caudate nucleus, ventral midbrain, bilateral anterior insula, bilateral dorsolateral PFC (DLPFC), the dorsal ACC (dACC), the bilateral TPJ, and the cerebellum. The opposite contrast (Control $>$ Pumps) revealed activation in the paracentral gyrus, bilateral inferior insula, ventromedial PFC, middle temporal gyrus, and middle frontal gyrus. No brain regions were significantly activated to cash outs or explosions.

Next we examined whether there were gender or age differences in neural activation on the BART. In wholebrain regression analyses, we simultaneously correlated gender (dummy-coded boys $=0$, girls $=1$ ) and age (in months) on brain activation on the BART. Age was not associated with brain activation during the BART. We did find a few gender differences, such that girls showed greater activation than boys in the bilateral cuneus and bilateral fusiform during cash outs, and boys showed greater activation than girls in the precuneus and primary sensory cortex (BA 3,1,2) during Pumps > Control (see Table 3A).

\section{Main Effects on the GNG}

In whole-brain analyses, we examined neural activation to successful response inhibitions (no-go trials). As shown in Table 2B and Figure 2B, successful response inhibitions (no-go $>$ go) activated brain regions involved in cognitive control, including the bilateral DLPFC and dACC. Other significant brain regions included the bilateral anterior insula, inferior parietal lobule, visual cortex, precuneus, and cerebellum. The opposite contrast (go $>$ no-go) revealed activation in the precuneus, premotor cortex, ventromedial PFC, STS, bilateral inferior insula, cerebellum, and fusiform gyrus,

Next we examined whether there were sex or age differences in neural activation on the GNG. In wholebrain regression analyses, we simultaneously correlated gender (dummy-coded boys $=0$, girls $=1$ ) and age (in months) on brain activation during no-go $>$ go trials. With age, participants showed greater activation in the right DLPFC, cuneus, precuneus, and posterior STS (see Table 3B). There were no sex differences in brain activation to no-go $>$ go.

\section{Neural Correlates of Family Obligation and Risk Taking}

Next, we examined how family obligation was related to neural regions involved in risk taking. In whole-brain regression analyses, we correlated family obligation values with neural activation during pumps, cash outs, and explosions. With increasing pumps (Pumps $>$ Control), family obligation values were positively related to activation in the bilateral fusiform (Table 4). Family obligation values were not negatively correlated with any brain regions during pumps. For Cash Outs, family obligation values were negatively correlated with activation in the bilateral VS as the number of pumps increased (see Figure 3 and Table 4). In other words, there was greater VS activation in cash-out trials that yielded greater reward value, and this activation was negatively associated with family obligation values. The link between adolescents' family obligation values and VS activation remains significant when controlling for the total number of red balloons and the total number of balloons cashed, suggesting that the number of balloons in the analysis are not driving the results. Family obligation values were also associated with decreased activation in the bilateral visual cortex to cash outs. No brain regions correlated positively with family obligation values during cash outs. Family obligation values did not relate to neural activation during explosions.

\section{Neural Correlates of Family Cohesion and Support and Risk Taking}

Next, we examined whether family cohesion and support show similar patterns as those found for family obligation values. In whole-brain regression analyses, we correlated family cohesion and support with neural activation to pumps, cash outs, and explosions. Family cohesion and support did not correlate with activation during any of these contrasts. Moreover, in whole-brain regression analyses controlling for family cohesion, family obligation continues to predict dampened bilateral VS activation above and beyond the effects of family cohesion and support $(Z=3.52, p<.05$, corrected).

\section{Neural Correlates of Family Obligation and Behavioral Inhibition}

Next, we examined how family obligation related to neural response during behavioral inhibition by examining activation to no-go > go trials on the GNG. In whole-brain regression analyses family obligation values were positively correlated with activation in the left DLPFC (see Figure 4 and Table 5). No brain regions correlated negatively with family obligation values.

\section{Neural Correlates of Family Cohesion and Support and Behavioral Inbibition}

We examined whether family cohesion and support show similar patterns as those observed for family obligation values. In whole-brain regression analyses, we correlated family cohesion and support with neural activation to successful behavioral inhibitions (no-go > go). Family cohesion and support did not correlate with neural activation during no-go $>$ go trials. In whole-brain regression analyses controlling for family cohesion and support, family 
Table 3. Neural Regions Activated during (A) Risk Taking and (B) Response Inhibition

\begin{tabular}{|c|c|c|c|c|c|c|}
\hline Contrast & Anatomical Region & $x$ & $y$ & $z$ & $\operatorname{Max} Z$ & $k$ \\
\hline \multicolumn{7}{|l|}{ (A) Risk Taking } \\
\hline \multirow[t]{13}{*}{ Pumps $>$ Control } & $\mathrm{R}$ anterior insula & 38 & 18 & 2 & 7.80 & $37,798^{\mathrm{a}}$ \\
\hline & $\mathrm{L}$ anterior insula & -36 & 18 & 6 & 6.20 & a \\
\hline & dACC & -2 & 20 & 38 & 7.41 & $\mathrm{a}$ \\
\hline & R VS & 19 & 8 & -6 & 4.50 & $\mathrm{a}$ \\
\hline & L VS & -14 & 6 & -4 & 4.07 & a \\
\hline & R DS & 16 & 4 & 14 & 4.06 & a \\
\hline & L DS & -18 & 6 & 12 & 4.02 & a \\
\hline & R DLPFC & 30 & 52 & 28 & 5.29 & a \\
\hline & L DLPFC & -30 & 48 & 20 & 4.81 & a \\
\hline & $\mathrm{R}$ cerebellum & 26 & -50 & -26 & 5.52 & $\mathrm{a}$ \\
\hline & L cerebellum & -36 & -56 & -34 & 5.69 & a \\
\hline & ventral midbrain & 2 & -18 & -16 & 5.48 & $\mathrm{a}$ \\
\hline & $\mathrm{R}$ inferior parietal cortex & 42 & -48 & 40 & 5.04 & 2,169 \\
\hline \multirow[t]{6}{*}{ Control > Pumps } & paracentral lobule & 22 & -28 & 72 & 6.20 & $31,325^{\mathrm{b}}$ \\
\hline & $\mathrm{R}$ inferior insula & 42 & -16 & 16 & 4.70 & b \\
\hline & $\mathrm{R}$ middle temporal gyrus & 44 & -80 & 16 & 4.50 & $\mathrm{~b}$ \\
\hline & L middle temporal gyrus & -50 & -64 & 20 & 4.58 & b \\
\hline & orbital frontal cortex & 6 & 24 & -14 & 5.89 & $3,326^{\mathrm{c}}$ \\
\hline & $\mathrm{L}$ middle frontal gyrus & -32 & 8 & 54 & 4.2 & c \\
\hline \multicolumn{7}{|c|}{ (B) Response Inbibition } \\
\hline \multirow[t]{11}{*}{ no-go $>$ go } & $\mathrm{R}$ anterior insula & 28 & 20 & -10 & 6.15 & $11,198^{\mathrm{d}}$ \\
\hline & R DLPFC & 36 & 48 & 22 & 3.89 & d \\
\hline & dACC & 6 & 30 & 28 & 4.37 & d \\
\hline & $\mathrm{R}$ inferior parietal cortex & 58 & -44 & 34 & 6.78 & 7,623 \\
\hline & L inferior parietal cortex & -60 & -38 & 28 & 6.36 & 3,463 \\
\hline & $\mathrm{L}$ anterior insula & -34 & 18 & 6 & 5.30 & 1,265 \\
\hline & L DLPFC & -32 & 52 & 16 & 4.09 & 857 \\
\hline & $\mathrm{R}$ visual cortex & 28 & -94 & -6 & 6.30 & 754 \\
\hline & $\mathrm{R}$ precuneus & 10 & -68 & 40 & 4.81 & 621 \\
\hline & L visual cortex & -30 & -96 & -6 & 5.37 & 577 \\
\hline & L cerebellum & -34 & -60 & -28 & 3.84 & 528 \\
\hline \multirow[t]{8}{*}{ go > no-go } & precuneus & -4 & -54 & 6 & 6.08 & $13,182^{\mathrm{e}}$ \\
\hline & premotor cortex & 0 & -36 & 58 & 4.2 & e \\
\hline & orbital frontal cortex & 2 & 40 & -20 & 6.06 & 6,580 \\
\hline & STS & -62 & -10 & -16 & 4.76 & $1,485^{\mathrm{f}}$ \\
\hline & $\mathrm{L}$ inferior insula & -48 & -14 & 16 & 3.82 & $\mathrm{f}$ \\
\hline & L cerebellum & 64 & -6 & 6 & 4.56 & 600 \\
\hline & $\mathrm{R}$ inferior insula & 14 & -88 & -38 & 5.06 & 483 \\
\hline & L fusiform gyrus & -54 & -48 & -14 & 4.28 & 461 \\
\hline
\end{tabular}

$\mathrm{L}$ and $\mathrm{R}$ refer to left and right hemispheres; $x y$ and $z$ refer to MNI coordinates; Max $Z$ refers to the $z$ score at those coordinates (local maxima); $k$ refers to the number of voxels in each significant cluster. Anatomical regions that share functional clusters are denoted with the same superscript letter. All regions are listed at cluster-forming threshold of $Z>2.3$ and an extent threshold of $p<.05$ corrected using the Theory of Gaussian Random Fields. DS = dorsal striatum. 
Table 4. Neural Regions that Correlated Negatively with Family Obligation Values during Cash-out Trials that Increased Parametrically According to the Amount of Reward

\begin{tabular}{lrrrrrr}
\hline Anatomical Region & $B A$ & \multicolumn{1}{c}{$x$} & \multicolumn{1}{c}{$y$} & $z$ & $\operatorname{Max} Z$ & $k$ \\
\hline Right VS & & 20 & 10 & -8 & 4.04 & 697 \\
Left VS & & -6 & 8 & 0 & 3.72 & 565 \\
Right visual cortex & $17 / 18$ & -20 & -104 & 2 & 3.79 & 38 \\
Left visual cortex & $17 / 18$ & 6 & -106 & 16 & 3.44 & 509 \\
\hline
\end{tabular}

$x, y$, and $z$ refer to MNI coordinates; Max $Z$ refers to the $z$ score at those coordinates (local maxima); $k$ refers to the number of voxels in each significant cluster. All regions are listed at cluster-forming threshold of $Z>$ 2.3 and an extent threshold of $p<.05$ corrected using the Theory of Gaussian Random Fields. BA refers to putative Brodman's area.

obligation continues to predict greater DLPFC activation during behavioral inhibitions above and beyond the effects of family cohesion and support $(Z=3.89, p<.05$, corrected).

\section{Linking Neural Activation to Real-life Risk Taking and Decision-making}

Finally, we examined how percent BOLD signal change in the VS to increasing rewards relates to adolescents' self-reported risk taking as assessed using the Adolescent Risk Taking Scale. We extracted the percent BOLD signal change in the VS to cash-out trials from the cluster that correlated with family obligation values and regressed it onto risk taking in SPSS. Controlling for age and gender, decreased VS activation to cash outs was associated with less risk-taking behavior (right VS: $B=1.50, S E=.49, \beta=$ $.43, p<.005$; left VS: $B=1.15, S E=.54, \beta=.32, p<.05$; Figure 5A).

Next we examined how percent BOLD signal change in the DLPFC to behavioral inhibitions relates to adolescents' self-report decision-making as assessed using the Flinders Adolescent Decision-making Questionnaire. We extracted percent BOLD signal change in the DLPFC to successful behavioral inhibitions (i.e., no-go $>$ go trials) from the cluster that correlated with family obligation and regressed it onto decision-making competence in SPSS. Controlling for age and sex, DLPFC activation to no-go $>$ go trials was associated with better decisionmaking competence $(B=5.50, S E=1.42, \beta=.53, p<$ .001 ; Figure 5B).

Lastly, we examined how self-reported risk taking relates to neural activation during the BART. In whole-brain regression analyses, risk taking was associated with increased activation in the left DLPFC (xyz =-3030 46, $z=3.77, k=898, p<.05$, corrected) and left VLPFC $(x y z=-1854-14, z=4.19, k=1418, p<.05$, corrected $)$ during Pumps $>$ Control. Risk taking was not associated with neural activation during cash outs at a whole-brain corrected threshold. Decision-making competence was

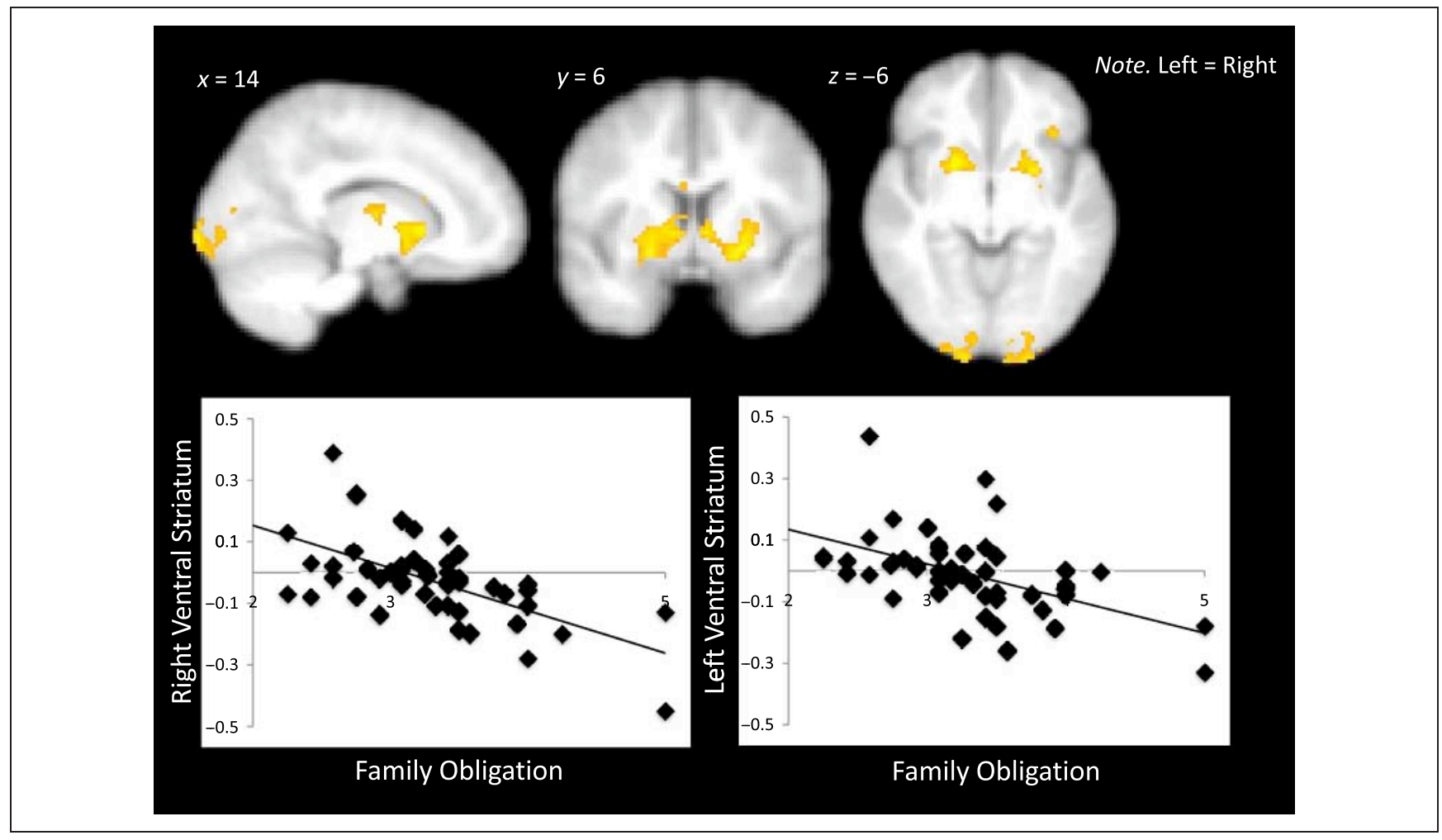

Figure 3. Neural regions that correlated negatively with family obligation values during cash outs. Percent BOLD signal change was extracted from the right and left VS during cash outs and plotted with family obligation values. 
Figure 4. Percent BOLD signal change in the DLPFC during behavioral inhibitions that correlated with family obligation values

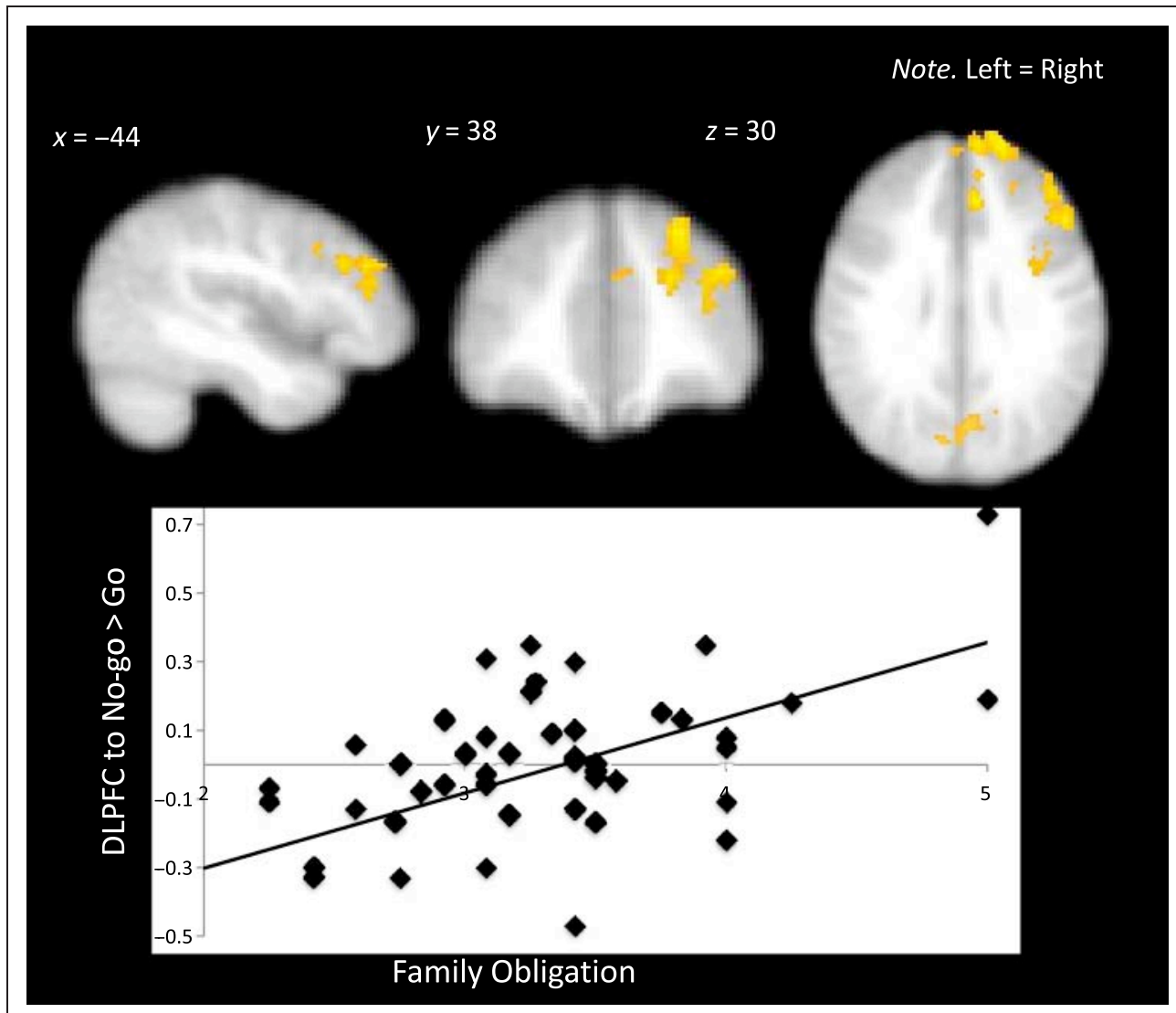

not associated with neural activation to no-go $>$ go at the whole-brain corrected threshold.

\section{DISCUSSION}

Vulnerability to risk taking during adolescence is normative and arises, in part, because of changes in the brain's neural circuitry. Discordant development of brain regions responsible for cognitive control and reward processing may render adolescents more susceptible to emotionally driven, reward-seeking behaviors and less able to modulate such decisions (Somerville et al., 2010; Steinberg, 2008). Previous reports have shown that increased family obligation

Table 5. Neural Regions that Correlated Positively with Family Obligation Values during Behavioral Inhibition on the GNG Task

\begin{tabular}{lcccccc}
\hline Anatomical Region & $B A$ & $x$ & $y$ & $z$ & $\operatorname{Max} Z$ & $k$ \\
\hline Left DLPFC & $9 / 46$ & -44 & 38 & 30 & 4.05 & 1943 \\
Left precuneus & 7 & -6 & -64 & 42 & 3.44 & 557 \\
\hline
\end{tabular}

$x, y$, and $z$ refer to MNI coordinates; Max $Z$ refers to the $z$ score at those coordinates (local maxima); $k$ refers to the number of voxels in each significant cluster. All regions are listed at cluster-forming threshold of $Z>2.3$ and an extent threshold of $p<.05$ corrected using the Theory of Gaussian Random Fields. BA refers to the putative Brodman's area. is associated with decreased risk taking (e.g., German et al., 2009; Ramirez \& de la Cruz, 2003; Kaplan et al., 2001; Gil et al., 2000), but the neural mechanism underlying this relationship remained elusive. Our results suggest that family obligation may alter activation in neural regions involved in reward sensitivity and cognitive control and activation in these neural regions is associated with adolescents' real-life risk-taking behaviors and decision-making skills.

Family obligation values were associated with decreased pumps on the BART, suggesting that adolescents with higher family obligation values have a lower risk preference. Behaviorally, more participants are willing to inflate the balloons during the BART, the greater risk level they are willing to take. Moreover, behavioral performance on the BART is associated with real-life risk taking (Bornovalova et al., 2009; Lejuez et al., 2003). Therefore, the average number of inflations represents an objective assessment of risk preference (Rao, Korczykowski, Pluta, Hoang, \& Detre, 2008). In addition, family obligation values were related to dampened activation in the VS to increasing monetary rewards. The VS is typically associated with reward sensitivity and approach-related behavior (Delgado, Nystrom, Fissell, Noll, \& Fiez, 2000; Knutson, Westdorp, Kaiser, \& Hommer, 2000). Together, these behavioral and neural results suggest that adolescents who value family obligation to a greater extent are less oriented to risky decisions (i.e., greater risk aversion) and have a lower 
Figure 5. Percent BOLD signal change in (A) the VS that correlated with self-reported risk taking and (B) the DLPFC that correlated with self-reported decision-making skills.

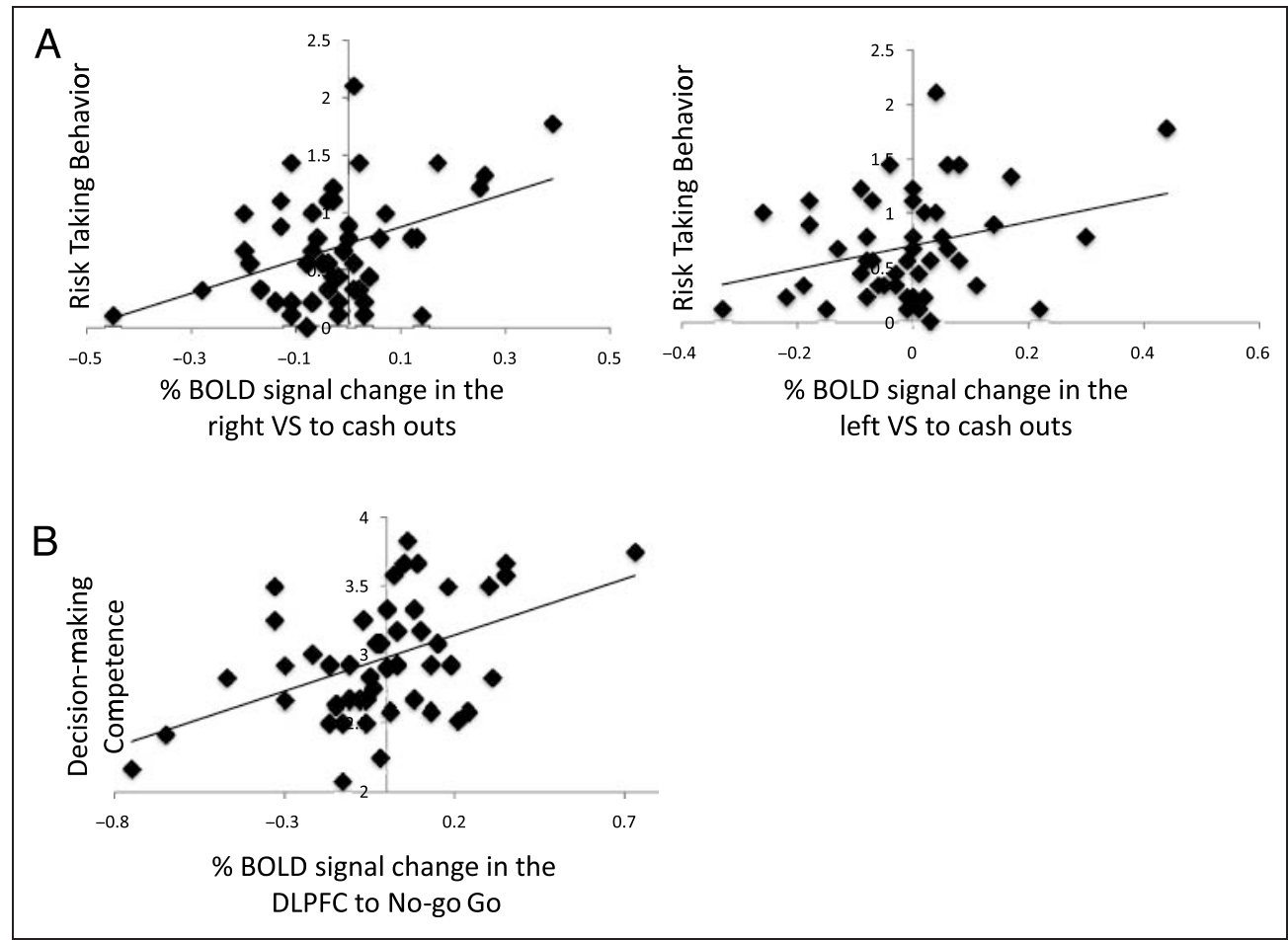

sensitivity to increasing rewards. It is possible that family obligation values decrease the affective salience of risk taking because of the motivation to avoid risk. Indeed, some prior work suggests that adolescent risk taking does not show the typical peaks in the absence of emotional arousal (Figner et al., 2009).

The task design of the BART precludes our ability to delineate the specific processes that may be involved during cash-out trials. These decisions may involve reward sensitivity, such that adolescents cash out to gain increasing monetary rewards, or these decisions may involve risk aversion, such that adolescents cash out to avoid an explosion and lose a potential monetary gain. Thus, the effects of risk and reward are confounded in this study. One neuroimaging study among adults using the BART (Schonberg et al., 2012) suggests that reward is constant across pumps whereas risk or loss increases with pumps, suggesting that individuals cash out after greater pumps to avoid a loss. Future studies should continue to attempt to dissociate how risk and reward are processed independently of one another and whether family obligation predicts risk aversion, reward sensitivity, or both.

Family obligation values were also associated with greater activation in the DLPFC during successful behavioral inhibition. Prior work has found that adults recruit the lateral PFC to a greater extent than adolescents during risk taking (Chein et al., 2010; Eshel et al., 2007), and children show decreased activation in the lateral PFC compared with adults when matched on performance during a cognitive control task (Bunge et al., 2002). In- deed, we found that heightened DLPFC activation was associated with better decision-making skills. Moreover, when we examined age effects during behavioral inhibition, older adolescents showed greater recruitment of the DLPFC. Together, this prior work (e.g., Eshel et al., 2007; Bunge et al., 2002) and the results of our study suggest that increased DLPFC activation is suggestive of more mature cognitive control. We speculate that, in our study, the more "mature" use of the DLPFC during the cognitive control task may render adolescents with higher family obligation better able to regulate emotionally driven behaviors, thereby reducing risk taking. However, other work has found that adults show more focal recruitment of the DLPFC, whereas adolescents show more diffuse activation during response inhibition (Durston et al., 2006). This work suggests that with practice and development, prefrontal activation actually decreases, reflecting a more automatic skill. An alternative explanation for the increased DLPFC activation in our study may be a greater motivation to recruit cognitive control efforts in the high family obligation individuals. Because staying out of trouble is an important aspect of adolescents' family obligations, motivation to control behavior in socially desirable ways may be higher among these adolescents, and so they engage the DLPFC to a greater degree. The cognitive control system is highly flexible during adolescence, and the degree to which adolescents engage in successful self-regulation may highly depend upon their motivation to do so (Crone \& Dahl, 2012).

Family obligation is a unique aspect of family relationships that relates to reduced risk-taking behavior above 
the effects of family cohesion and support. Individuals who more strongly value helping others and putting others' needs before their own are more likely to assign priority to another's welfare and regulate their own behavior and emotions to meet another's needs (Caprara \& Steca, 2007). Indeed, adolescents who value family obligation often put the needs of their family before their own, consider the needs of their family when making important life decisions, and think about their family's needs and wishes for the future (Fuligni et al., 1999). Moreover, decisions to assist one's family recruit neural regions involved in cognitive control (Telzer et al., 2011). Thus, family obligation provides adolescents opportunities to engage in and practice self-control. In addition, adolescents who value family obligation report more negative consequences for engaging in risky behavior (German et al., 2009), and so these adolescents may find risk taking to be comparatively less rewarding. We found that family cohesion and support itself was not associated with neural activation during risk taking or cognitive control, suggesting that it is not simply about having a close and supportive family that reduces neural sensitivity to risk. Rather, the independent predictive value of family obligation suggests the results are because of specific types of family relationships that foster self-regulatory skills and an avoidance of behaviors that could have negative consequences. Therefore, it is the affective and motivational aspects (such as feelings of value, meaningfulness, and intrinsic reward) that underpin these effects and not simply the benefits of a close and supportive family.

If dampened VS activation during the BART is suggestive of reduced reward sensitivity and greater risk aversion and increased DLPFC activation during the GNG is suggestive of better decision-making skills, these neural activations should also be associated with adolescents' real-life risk taking and decision-making. Indeed, we found that dampened VS activation is associated with less selfreported risk-taking behaviors. In addition, adolescents who show greater DLPFC activation report better decisionmaking competence, corroborating that enhanced DLPFC activation is indicative of more mature use of this region, relating to better decision-making skills. These findings support the notion that family obligation may reduce neural sensitivity to risk-taking behavior and are consistent with other studies showing that heightened DLPFC and dampened VS activation are associated with lower risktaking behaviors in adolescents (Eshel et al., 2007; Galvan et al., 2007; Ernst et al., 2005).

Although not the primary purpose of this study, we found that increasing risk during the BART (i.e., greater pumps) was associated with robust neural activity in the mesolimbic-frontal pathway, including the striatum, ventral midbrain, insula, dACC, and DLPFC. These activations are consistent with those of Schonberg and colleagues (2012) and Rao and colleagues (2008), who used a modified version of the BART among adults. Interestingly, explosions and cash outs did not recruit any brain re- gions in the main effects. Perhaps this is because these contrasts are modulated by individual differences. Indeed, neural response to the cash-out trials was modulated by family obligation, such that only individuals with the lowest values showed heightened activation in the VS. Future research should attempt to understand other individual differences among adolescents that might predict neural sensitivity to punishment (explosions) and rewards (cash outs).

We examined an aspect of family life that is culturally relevant to Mexican families. By taking this approach, we were able to identify a "cultural resource" for these adolescents, identifying how family obligation can be a protective factor. We believe these findings apply to youth from diverse cultural backgrounds. By engaging in social relationships that allow adolescents to put the needs of others before their own, by engaging in self-control, and attaining a sense of meaning from the relationship, adolescents may develop the skills and motivations needed to avoid risk taking. Future research should examine how other social relationships, such as participating in community service, engaging in positive peer relationships such as academic clubs and religious engagement can similarly reduce risk taking among diverse adolescents.

\section{Acknowledgments}

Support for this study was provided by the NICHD (R01HD057164-S and R01HD057164; Fuligni), the Center for Culture, Brain and Development Research Grant (Fuligni and Galvan), an NSF Doctoral Dissertation Improvement Grant (Telzer), an SRCD Dissertation Fund Award (Telzer), an APF and COGDOP Graduate Research Grant (Telzer), and a University of California Institute for Mexico and the United States Dissertation Research Grant (Telzer), Preparation of this manuscript was supported in part by a National Research Service Award Graduate Fellowship (Telzer).

Reprint requests should be sent to Eva H. Telzer, Department of Psychology, University of Illinois, 603 East Daniel Street, Champaign, IL 61820, or via e-mail: ehtelzer@illinois.edu.

\section{REFERENCES}

Alexander, C. S., Kim, Y. J., Ensminger, M., Johnson, K. E., Smith, B. J., \& Dolan, L. J. (1990). A measure of risk taking for young adolescents: Reliability and validity assessments. Journal of Youth and Adolescence, 19, 559-569.

Armsden, G. C., \& Greenberg, M. T. (1987). The inventory of parent and peer attachment: Individual differences and their relationship to psychological well-being in adolescence. Journal of Youth and Adolescence, 16, 427-445.

Arnett, J. J. (1992). Reckless behavior in adolescence: A developmental perspective. Developmental Review, 12, 339-373.

Beckmann, C., Jenkinson, M., \& Smith, S. (2003). General multilevel linear modeling for group analysis in fMRI. Neuroimage, 20, 1052-1063.

Bjork, J. M., Smith, A. R., Chen, G., \& Hommer, D. W. (2010). Adolescents, adults and rewards: Comparing motivational neurocircuitry recruitment using fMRI. PLoS One, 5, e11440.

Bornovalova, M. A., Cashman-Rolls, A., O’Donnell, J. M., Ettinger, K., Richards, J. B., Dewit, H., et al. (2009). Risk taking differences on a behavioral task as a function of 
potential reward/loss magnitude and individual differences in impulsivity and sensation seeking. Pharmacology Biochemistry and Behavior, 34, 685-692.

Bunge, S. A., Dudukovic, N. M., Thomason, M. E., Vaidya, C. J., \& Gabrieli, J. D. E. (2002). Immature frontal love contributions to cognitive control in children: Evidence from fMRI. Neuron, 33, 301-311.

Caprara, G. V., \& Steca, P. (2007). Prosocial agency: The contribution of values and self-efficacy beliefs to prosocial behavior across ages. Journal of Social and Clinical Psychology, 26, 220-241.

Casey, B. J., Getz, S., \& Galvan, A. (2008). The adolescent brain. Developmental Review, 28, 62-77.

Chambers, R., Taylor, J., \& Potenza, M. (2003). Developmental neurocircuitry of motivation in adolescence: A critical period of addiction vulnerability. American Journal of Psychiatry, 160, 1041-1052.

Chein, J., Albertm, D., O’Brien, L., Uckert, K., \& Steinberg, L. (2010). Peers increase adolescent risk taking by enhancing activity in the brain's reward circuitry. Developmental Science, 14, F1-F10.

Crone, E. A., \& Dahl, R. E. (2012). Understanding adolescence as a period of social-affective engagement and goal flexibility. Nature Reviews, 13, 636-650.

Cuellar, I., Arnold, B., \& Gonzalez, G. (1995). Cognitive referents of acculturation: Assessment of cultural constructs in Mexican Americans. Journal of Community Psychology, 23, 339-356.

Dahl, R. E. (2004). Adolescent brain development: A period of vulnerabilities and opportunities. Annals of the New York Academy of Sciences, 1021, 1-22.

de la Rosa, M. R. (1988). Natural support systems of Puerto Ricans: A key dimension for their well-being. Health Social Work, 15, 181-190.

Delgado, M. R., Nystrom, L. E., Fissell, C., Noll, D. C., \& Fiez, J. A. (2000). Tracking the hemodynamic responses to reward and punishment in the striatum. Journal of Neurophysiology, 84, 3072-3077.

Durston, S., Davidson, M. C., Tottenham, N., Galvan, A., Spicer, J., Fossella, J. A., et al. (2006). A shift from diffuse to focal cortical activity with development. Developmental Science, 9, 1-8.

Ernst, M., Nelson, E. E., Jazbec, S., McClure, E. B., Monk, C. S., Leibenluft, E., et al. (2005). Amygdala and nucleus accumbens in responses to receipt and omission of gains in adults and adolescents. Neuroimage, 25, $1279-1291$.

Eshel, N., Nelson, E. E., Blair, R. J., Pine, D. S., \& Ernst, M. (2007). Neural substrates of choice selection in adults and adolescents: Development of the ventrolateral prefrontal and anterior cingulate cortices. Neuropsychologia, 45, 1270-1279.

Figner, B., Mackinlay, R. J., Wilkening, F., \& Weber, E. U. (2009). Affective and deliberative processes in risky choice: Age difference in risk taking in the Columbia Card Task. Journal of Experimental Psychology, 35, 709-730.

Fuligni, A. J. (2001). Family obligation and the academic motivation of adolescents from Asian, Latin American, and European backgrounds. In A. Fuligni (Ed.), Family obligation and assistance during adolescence: Contextual variations and developmental implications (New directions in child and adolescent development monograph) (pp. 61-76). San Francisco: Jossey-Bass, Inc.

Fuligni, A. J., Tseng, V., \& Lam, M. (1999). Attitudes toward family obligations among American adolescents from Asian, Latin American, and European backgrounds. Child Development, 70, 1030-1044.
Galvan, A., Hare, T. A., Parra, C. E., Penn, J., Voss, H., Glover, G., et al. (2006). Earlier development of the accumbens relative to orbitofrontal cortex might underlie risk-taking behavior in adolescents. Journal of Neuroscience, 26, 6885-6892.

Galvan, A., Hare, T., Voss, H., Glover, G., \& Casey, B. J. (2007). Risk-taking and the adolescent brain: Who is at risk? Developmental Science, 10, F8-F14.

García Coll, C., \& Vázquez García, H. A. (1995). Hispanic children and their families: On a different track from the very beginning. In $\mathrm{H}$. Fitzgerald, B. Lester, \& B. Zuckerman (Eds.), Children of poverty: Research, health, and policy issues (pp. 57-83). New York: Garland Publishing.

Gardner, M., \& Steinberg, L. (2005). Peer influence on risk-taking, risk preference, and risky decision-making in adolescence and adulthood: An experimental study. Developmental Psychology, 41, 625-635.

German, M., Gonzales, N. A., \& Dumka, L. (2009). Familism values as a protective factor for Mexican-origin adolescents exposed to deviant peers. The Journal of Early Adolescence, 29, $16-42$

Gfoerer, J., \& de la Rosa, M. (1993). Protective and risk factors associated with drug use among Hispanic youth. Journal of Addictive Diseases, 12, 87-107.

Gil, A. G., Wagner, E. F., \& Vega, W. A. (2000). Acculturation, familism, and alcohol use among Latino adolescent males: Longitudinal relations. Journal of Community Psychology, 28, 443-458.

Jenkinson, M., Bannister, P., Brady, M., \& Smith, S. (2002). Improved optimization for the robust and accurate linear registration and motion correction of brain images. Neuroimage, 17, 825-841.

Jolles, D. D., van Buchem, M. A., Rombouts, S. A., \& Crone, E. A. (2012). Practice effects in the developing brain: A pilot study. Developmental Cognitive Neuroscience, 2, 180-191.

Kaplan, C. P., Napoles-Springer, A., Stewart, S. L., \& Perez-Stable, E. J. (2001). Smoking acquisition among adolescents and young Latinas: The role of socioenvironmental and personal factors. Addictive Behaviors, 26, 531-550.

Knutson, B., Westdorp, A., Kaiser, E., \& Hommer, D. (2000). fMRI visualization of brain activity during a monetary incentive delay task. Neuroimage, 12, 20-27.

Lejuez, C. W., Aklin, W. M., Zvolensky, M. J., \& Pedulla, C. M. (2003). Evaluation of the Balloon Analogue Risk Task (BART) as a predictor of adolescent real-world risk-taking behaviours. Journal of Adolescence, 26, 320-345.

Lejuez, C. W., Read, J. P., Kahler, C. W., Richards, J. B., Ramsey, S. E., Stuart, G. L., et al. (2002). Evaluation of a behavior measure of risk taking: The Balloon Analogue Risk Task BART. Journal of Experimental Psychology: Applied, 8, 75-84.

Luna, B., Padmanabhan, A., \& O'Hearn, K. (2010). What has fMRI told us about the development of cognitive control through adolescence? Brain and Cognition, 72, 101-113.

Mann, L., Harmoni, R., \& Power, C. (1989). Adolescent decision-making: The development of competence. Journal of Adolescence, 12, 265-278.

Miniño, A. M. (2010). Mortality among teenagers aged 12-19 years: United States, 1999-2006. NCHS Data Brief, no 37. Hyattsville, MD: National Center for Human Statistics.

Pfiefer, J. H., \& Allen, N. B. (2012). Arrested development? Reconsidering dual-systems models of brain function in adolescence and disorders. Trends in Cognitive Sciences, 16, 322-329.

Poline, J.-B., Worsley, K. J., Evans, A., \& Friston, K. (1997). Combining spatial extent and peak intensity to test for activations in functional imaging. Neuroimage, 5, 83-96.

Ramirez, R. R., \& de la Cruz, G. P. (2003). The Hispanic population in the United States: March 2002, Current 
Population Reports, P20-545. Washington: U.S. Census Bureau.

Rao, H., Korczykowski, M., Pluta, J., Hoang, A., \& Detre, J. A. (2008). Neural correlates of voluntary and involuntary risk taking in the human brain: An fMRI study of the Balloon Analog Risk Task (BART). Neuroimage, 42, 902-910.

Romero, A. J., \& Ruiz, M. (2007). Does familism lead to increased parental monitoring?: Protective factors for coping with risky behaviors. Journal of Child and Family Studies, 16, 143-154.

Schonberg, T., Fox, C. R., Mumford, J. A., Congdon, E., Trepel, C., \& Poldrack, R. A. (2012). Decreasing ventromedial prefrontal cortex activity during sequential risk-taking: An fMRI investigation of the Balloon Analogue Risk Task. Frontiers in Decision Neuroscience, 6, 1-11.

Somerville, L. H., Jones, R. M., \& Casey, B. J. (2010). A time of change: Behavioral and neural correlates of adolescent sensitivity to appetitive and aversive environmental cues. Brain and Cognition, 72, 124-133.

Steinberg, L. (2008). A social neuroscience perspective on adolescent risk taking. Developmental Review, 28, 78-106.

Suárez-Orozco, C., \& Suárez-Orozco, M. M. (1995).

Transformations: Immigration, family life, and achievement motivation among Latino adolescents. Stanford, CA: Stanford University Press.

Telzer, E. H., \& Fuligni, A. J. (2009). Daily family assistance and the psychological well being of adolescents from Latin American, Asian, and European backgrounds. Developmental Psychology, 45, 1177-1189.
Telzer, E. H., Masten, C. L., Berkman, E., Lieberman, M. D., \& Fuligni, A. J. (2010). Gaining while giving: An fMRI investigation of the rewards of family assistance among White and Latino adolescents. Social Neuroscience, 5, 508-518.

Telzer, E. H., Masten, C. L., Berkman, E., Lieberman, M. D., \& Fuligni, A. J. (2011). Neural regions involved in self-control and mentalizing are recruited during prosocial decisions towards the family. Neuroimage, 58, 242-249.

Unger, J. B., Ritt-Olson, A., Teran, L., Huang, T., Hoffman, B. R., \& Palmer, P. (2002). Cultural values and substance use in a multiethnic sample of California adolescents. Addiction Research and Theory, 10, 257-279.

Vega, W. A., Zimmerman, R. S., Warheit, G. J., Apospori, E., \& Gil, A. G. (1993). Risk factors for early adolescent drug use in four ethnic and racial groups. American Journal of Public Health, 83, 185-189.

Warner, L. A., Valdez, A., Vega, W. A., de la Rosa, M., Turner, R. J., \& Canino, G. (2006). Hispanic drug abuse in an evolving cultural context: An agenda for research. Drug and Alcohol Dependence, 1(84 Suppl.), S8-S16.

Weisner, T. S., Matheson, C., Coots, J., \& Bernheimer, L. P. (2005). Sustainability of daily routines as a family outcome. In A. Maynard \& M. Martini (Eds.), Learning in cultural context: Family, peers and school (pp. 47-74). New York: Kluwer/Plenum.

Woolrich, M. (2008). Robust group analysis using outlier inference. Neuroimage, 41, 286-301. 